Allgemein erweist es sich als zweckmäßig, die "Subtilität" eines Effektes quantitativ durch die Größe

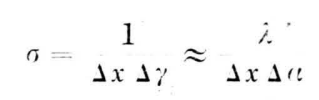

begrifflich zu fassen. Ein Effekt kann also nur mit einer Strahlkennzeichnung hinreichend großer Schärfe $g \geqq 0$ gemessen werden. $g / \sigma=\mu$ heiße „Meßbarkeit“; ist sie 1, so kann der Effekt mit der betreffen- den Strahlkennzeichnung noch auf etwa 10 bis $20 \%$ genau gemessen werden. Die Nachweisgrenze liegt bei etwa $\mu=0,2$. Wegen der Invarianz von $g, \sigma$ und $\mu$ gegen Abbildungen helfen diese Begriffe in verwickelten Fällen prinzipielle Fragen nach der M€ßbarkeit subtiler Effekte unmittelbar beantworten.

Hrn. Prof. Dr. Lochte-Holtgreven und Hrn. Prof. Dr. Uns öld, die die Wiederaufnahme dieser Arbeit ermöglichten und sie in jeder Weise unterstützten, möchte der Verf. an dieser Stelle danken.

\title{
Untersuchungen zur Strahlversetzung bei Totalreflexion des Lichtes mit der Methode der Minimumstrahlkennzeichnung*
}

\author{
Von Hans Wolter \\ Aus dem Institut für Experimentalphysik der Universität Kiel \\ (Z. Naturforschg. 5 a, 143-153 [1950]; eingegangen am 5. April 1949)
}

\begin{abstract}
Mit der Methode der Minimumstrahlkennzeichnung wurde die Strahlversetzung bei Totalreflexion gemessen. Experimente und Theorie stimmen hier auch bezüglich der starken Polarisationsabhängigkeit überein, im Gegensatz zu dem früheren Befund von G o o s und Hän che n ${ }^{1}$. Messungen mit einer verbesserten Maximummethode zeigten ebenfalls (freilich ungenauer) diєse Polarisationsabhängigkeit. Strahlversetzung wurde auch bei Reflexion an absorbierenden Medien festgestellt; sie wechselt hier meist mit der Polarisationsrichtung ihr Vorzeichen. Eine ausführliche Zusammenfassung befindet sich am Schluß.
\end{abstract}

\section{I.}

G oos und Hänchen ${ }^{1}$ fanden folgenden Effekt, für den hier die Bezeichnung Goos-HänchenEffekt vorgeschlagen sei. Ein durch zwei hintereinandergestellte Spalten ausgeblendeter Lichtstrahl wird bei Totalreflexion nicht an der Stelle der Grenze Glas-Luft, auf die er auftritt, reflektiert, sondern um eine kleine Strecke so parallel verschoben, als ob er einen Umweg durch das dünnere Medium gemacht hätte. Der Effekt liegt in der Größenordnung einer Wellenlänge; Goos und Hänchen vergrößerten ihn durch 34-fache Wiederholung der Reflexion und konnten die Verschiebung nach ihren eigenen Angaben auf $2 \%$ genau messen. Innerhalb dieser Grenzen fanden sie keine Abhängigkeit von der Polarisation.

Das widersprach unveröffentlichten Rechnungen des Verf., die zur Erklärung einer ähnlichen Versetzung bei elektromagnetischen Längstwellen dienten und infolge ihres sehr allgemeinen Ansatzes leicht auf die Totalreflexion spezialisiert werden konnten.

1 F. Go os u. H. Hänchen, Ann. Physik (6) 1, 333 [1947].

2 K. A rtm a n n, Ann. Physik (6) 2, 87 [1948].
Sie werden hier in II den Ausgangspunkt dieser Arbeit bilden. Die darauf aufbauenden experimentellen und theoretischen Untersuchungen haben auf zwei Fragen eine neue Antwort gegeben. Die erste behandelt die Abhängigkeit der Strahlversetzung in dichteren Medium vom Einfallswinkel und von der Polarisation; sie ist Gegenstand dieser Arbeit. Die zweite fragt nach den Vorgängen im dünneren Medium und an der Grenzfläche - also insbesondere nach dem "Umweg des Lichtes" — und wird in einer später zu veröffentlichenden Arbeit beantwortet.

Zur Klärung der ersten Frage trug inzwischen Artmann² wesentlich bei, der eine Theorie des Goos-Hänchen-Effekts gab (unter genauer Nachbildung der bei Goos und Hänchen herrschenden Verhältnisse) und eine Polarisationsabhängigkeit ausrechnete. Nach einer Betrachtung über mögliche Fehlerquellen, die ausgeschlossen werden konnten, vermutete Artmann aber schließlich die Ursache der Diskrepanz zwischen Theorie und Experiment in seiner Näherung, die statt der mathematisch zu schwie-

* Vorgetragen auf der Tagung der Deutschen Physikalischen Gesellschaft in Hamburg am 23. April 1949. 
rigen strengen Lösung den Kirchhoffschen Ansatz benutzte. Dieser steht in dem Ruf ${ }^{3}$, gerade bezüglich der Polarisationsverhältnisse nicht die richtigen Werte zu liefern.

Zur Klärung der Frage nach der Polarisationsabhängigkeit wird hier die Methode der Minimumstrahlkennzeichnung benutzt werden, wie sie in der unmittelbar vorhergehenden Arbeit allgemein beschrieben wurde. Diese gibt dem Experiment die erforderliche hohe Genauigkeit zur Untersuchung einer
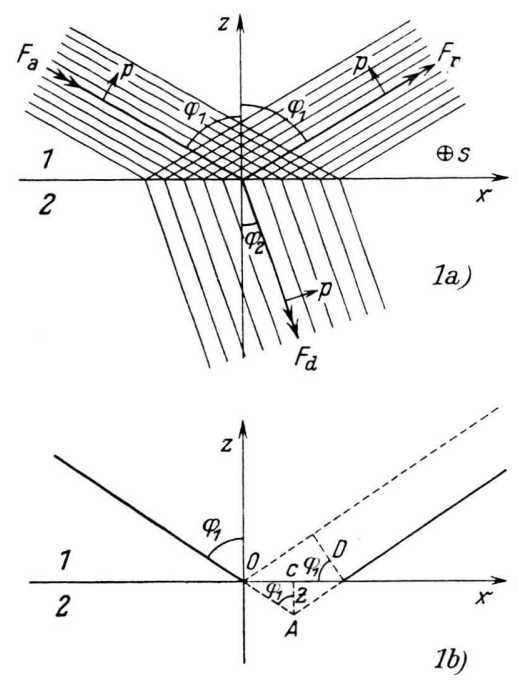

Abb. 1. Zur Erklärung der Bezeichnungen und des Koordinatensystems. a: Ebene Wellen an einer Grenzfläche. b: Zusammenhang zwischen Minimumversetzung $D$ und scheinbarer Reflexionstiefe $\mathrm{Z}$.

so subtilen Erscheinung und andererseits der Theorie die Einfachheit und Strenge, die an den Ergebnissen nicht zweifeln läßt.

In III werden außer totalreflektierenden auch andere Grenzflächen betrachtet werden. Erstens gewinnt das Problem durch Verallgemeinerung an Übersichtlichkeit; es ist zum Verständnis des Effekts wesentlich, daß er nicht auf Totalreflexion beschränkt ist. Zweitens wird unter den Fehlerquellen unserer Experimente auch eine Grenzfläche Glas-Silber eine Rolle spielen; wenn bei Goos und Hänchen wohl als evident galt, daß die Reflexion hierbei höchstens aus

3 Siehe u. a. G. Joos, Lehrbuch der theoretischen Physik, 6. Aufl., S. 336, Leipzig 1945. Dennoch trifft die Artmannsche Theorie die tatsächlichen Verhältnisse schon recht gut, wie unten noch diskutiert werden soll. Natürlich können Polarisationsabhängigkeiten durch den Kirchhoffschen Ansatz nicht richtig dargestellt werden, wenn sie durch die Spaltbacken verursacht werden. Doch hier stammen sie aus der Grenzfläche selbst und werden durch den Kirchhoffschen Ansatz nicht wesentlich verfälscht. einer "Tiefe" kleiner als die Silberschichtdicke kommen könne, so müssen wir uns doch bei unserer Auffassung über die Frage nach dem Weg des Lichtes von Begründungen aus der Anschauung freimachen.

II. Allgemeine Grundlagen der Theorie

Eine von zwei entfernten kohärenten gegenphasigen Quellen ausgehende elektromagnetische Welle soll der Einfachheit halber mathematisch erfaßt werden als Überlagerung zweier ebener Wellen, deren Fortpflanzungsrichtungen den kleinen Winkel $\Delta \ll 1$ einschließen und die unter den Einfallswinkeln $\varphi_{1}$ und $\varphi_{1}^{\prime}=\varphi-\Delta$ auf eine ebene Grenzfläche zwischen zwei Medien (1 und 2) mit den Brechungsindizes $n_{1}$ reell und $n_{2}$ komplex auffallen. Abb. 1 zeigt die Verhältnisse für eine der beiden Wellen. Unter $F_{\mathrm{a}}, F_{\mathrm{r}}, F_{\mathrm{d}}$ seien bei senkrechter Polarisation (〔 $\perp$ Einfallsebene) die elektrischen, bei paralleler Polarisation die magnetischen Feldstärken verstanden; diese zeigen dann in jedem Falle in die $y$-Richtung und haben für die einfallende erste bzw. zweite Welle die Ortsabhängigkeit

$$
\begin{gathered}
F_{\mathrm{a}}(x, y, z)=A_{0} \exp \left[-i \frac{2 \cdot \pi}{\lambda} n_{1}\left(x \sin \varphi_{1}-z \cos \varphi_{1}\right)\right], \\
F_{\mathrm{a}^{\prime}}(x, y, z)=A_{0}{ }^{\prime} \exp \left[-i \frac{2 \pi}{\lambda} n_{1}\left(x \sin \varphi_{1}{ }^{\prime}-z \cos \varphi_{1}{ }^{\prime}\right)\right] .
\end{gathered}
$$

Die Gegenphasigkeit erfassen wir (zugleich unter willkürlicher Verfügung über die hier uninteressante einfallende Intensität) durch $A_{0}=e^{i \omega t} ; A_{0}{ }^{\prime}=-e^{i \omega t}$. Die beiden einfallenden Wellen ergeben dann zusammen einen Feldstärkenbetrag

$$
\begin{array}{r}
F_{\mathrm{a}}+F_{\mathrm{a}^{\prime}}=2 \sin \left\{\tau _ { i } { } _ { i } { } _ { \mathrm { i } } \left(x\left(\sin \varphi_{1}-\sin \varphi_{1}{ }^{\prime}\right)\right.\right. \\
\left.\left.-z\left(\cos \varphi_{1}-\cos \varphi_{1}{ }^{\prime}\right)\right)\right\} .
\end{array}
$$

Für $\Delta \ll 1$ ist in zulässiger Näherung unter Benutzung der Abkürzung

$$
\begin{gathered}
\varphi_{1}=\frac{\varphi_{1}+\varphi_{1}{ }^{\prime}}{2} ; \\
F_{\mathrm{a}}+F_{\mathrm{a}^{\prime}}=2 \sin \left\{\begin{array}{c}
n_{1} \\
i
\end{array}\left(x \cos \varphi_{1}+z \sin \varphi_{1}\right) \Delta\right\} .
\end{gathered}
$$

Die einfallende Welle ist also durch ein Verschwinden der resultierenden Feldstärke innerhalb der Ursprungsebene

$$
x \cos \psi_{1}+\approx \sin \varphi_{1}=0
$$




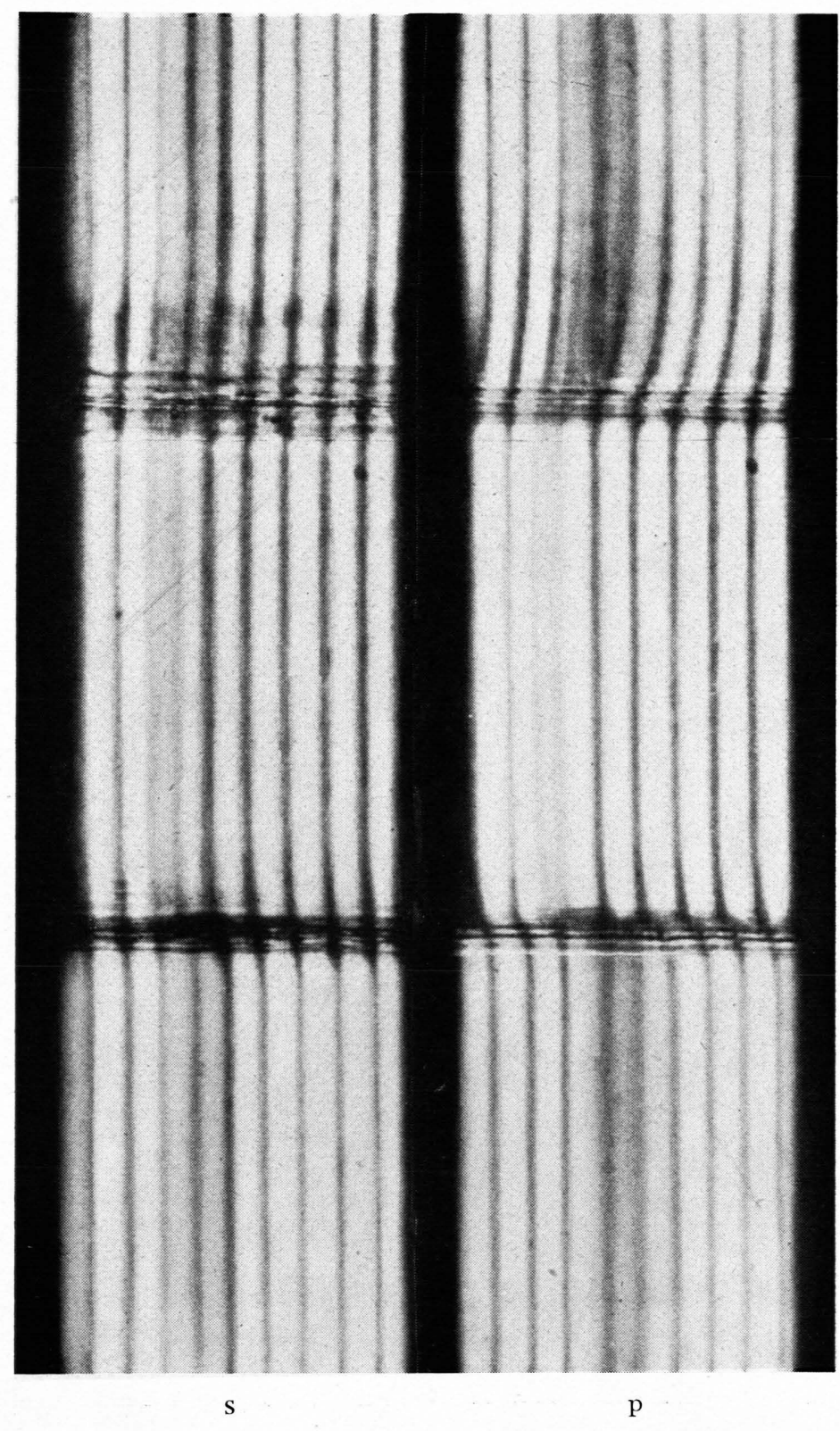

Abb. 4. Strahlversetzung des an der Grenze Glas - Luft (oben und unten) reflektierten Lichtes im Vergleich zur Reflexion an der Grenze Glas-Silber (Mitte), aufgenommen mit der Minimumstrahlkennzeichnung; $\alpha^{\prime}=$ Einfallswinkel - Grenzwinkel ist für die Minima jeweils von rechts beginnend: $1,35 \cdot 10^{-3} ; 0,95 \cdot 10^{-3} ; 0,55 \cdot 10^{-3} ; 0,2 \cdot 10^{-3}$; $-0,2 \cdot 10^{-3}$ Radien. 


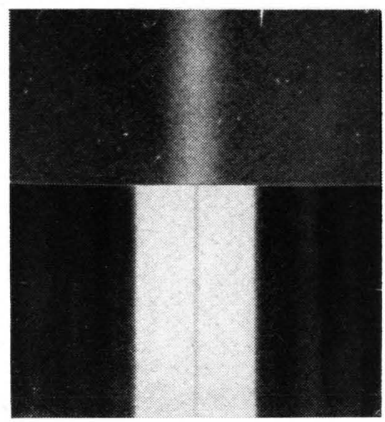

Abb. 1. Oben: Üblicher Lichtstrahl (Energiestrahl); unten: Strahlkennzeichnung mit einem Minimum optimaler Flankensteilheit. (Durchlaßöffnung, Aufnahmeabstand und Vergrößerung waren in beiden Fällen gleich.)

H.Wolter, Untersuchungen zur Strahlversetzung bei Totalreflexion des Lichtes. (S. 143)

a

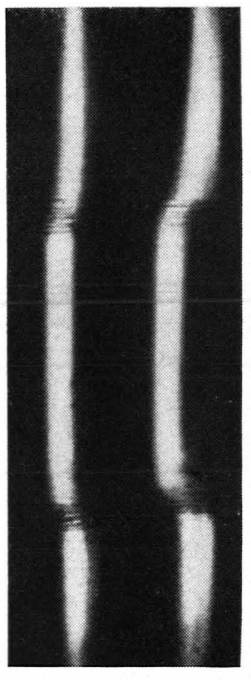

$\mathrm{b}$

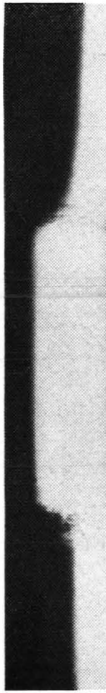

$\mathrm{p}$

S

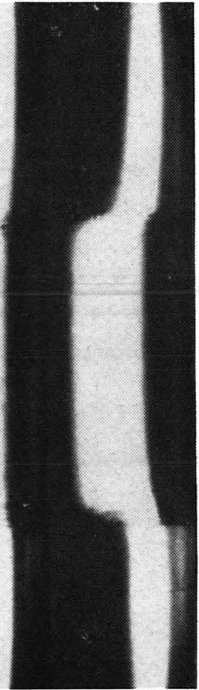

$\mathrm{p}$

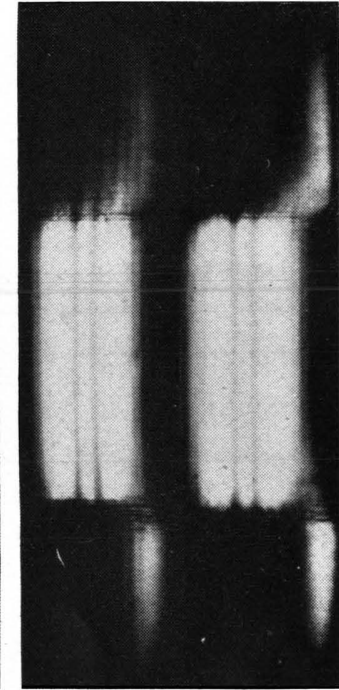

S

$\mathrm{p}$ $\mathrm{c}$

Abb. 9. Strahlversetzung bei Totalreflexion nach der Maximummethode, a mit optimalem, b mit schmälerem, c mit breiterem Spalt als strahldefinierendem Gebilde.

O.-E.Schweckendiek, Über eine Beobachtungskammer für ultramikroskopische Untersuchungen an Aerosolen. (S. 15.3)

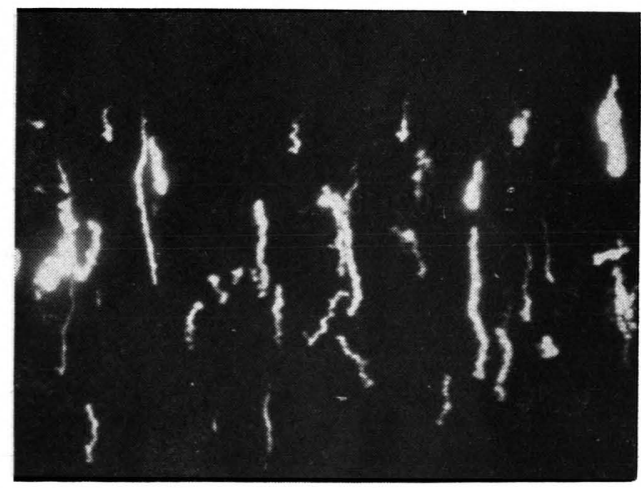

Abb. 3. Originalaufnahme von Fallstrecken von Aerosolteilchen im Schwerefeld. 
gekennzeichnet. Für die reflektierte Welle erhalten wir entsprechend

$$
\begin{aligned}
F_{\mathrm{r}}+F_{\mathrm{r}^{\prime}} & =\mathfrak{r} \exp \left[-\frac{2 i \pi}{i} n_{1}\left(x \sin \varphi_{1}+z \cos \varphi_{1}\right)\right] \\
& -\mathfrak{r}^{\prime} \exp \left[-\frac{2 \pi i}{i} n_{1}\left(x \sin \varphi_{1}^{\prime}+z \cos \varphi_{1}^{\prime}\right)\right] ;
\end{aligned}
$$

darin bedeuten $\mathfrak{x}$ und $\mathfrak{r}^{\prime}$ die Fresnelschen Reflexionskoeffizienten, die bekanntlich je nach Polarisationsrichtung verschiedene Werte $\mathfrak{r}_{s}, r_{s}{ }^{\prime}$ und $\mathfrak{r}_{p}, \mathfrak{r}_{p}{ }^{\prime}$ haben. Bezeichnen wir mit $\overline{\mathfrak{r}}$ das arithmetische Mittel aus $\mathfrak{r}$ und $r^{\prime}$, so erhalten wir nach leichter Umrechnung

$$
F_{\mathrm{r}}+F_{\mathrm{r}^{\prime}}=-\mathfrak{D}+\left(x \cos \varphi_{1}-z \sin \overline{\varphi_{1}}\right) \overline{\mathrm{r}} \Delta \frac{2 \pi n_{1}}{i}
$$

mit der Abkürzung

$$
\mathfrak{D}=\frac{i}{i, \pi n_{1}} \frac{\mathfrak{r}-\mathfrak{r}^{\prime}}{\mathfrak{r}+\mathfrak{r}^{\prime}} \frac{1}{\Delta}
$$

Der erste Faktor der rechten Seite von (6) ist

$$
\sqrt{\left(x \cos \varphi_{1}-z \sin \varphi_{1}-\operatorname{Re} \mathfrak{D}\right)^{2}+(\operatorname{Im} \mathfrak{D})^{2}}
$$

da von den Koordinaten nur der erste Summand unter der Wurzel abhängt, hat die reflektierte Welle also offenbar ein Minimum für

$$
x \cos \overline{\varphi_{1}}-z \sin \varphi_{1}-\operatorname{Re} \mathfrak{D}=0,
$$

d. h. in einer Ebene, die um das Stück

$$
\mathrm{D}=\operatorname{Re} \mathfrak{D}
$$

parallel verschoben ist gegenüber der normalerweise zu erwartenden Ebene $x \cos \bar{\varphi}_{1}-z \sin \bar{\varphi}_{1}=0$, wie Abb. 1 das andeutet. Re I entspricht also der „Strahlversetzung" im Sinne von Goos und Hänchen. Der Imaginärteil $\operatorname{Im} \mathfrak{I}$ ist ein $\mathrm{Ma}$ für die „Verschmierung" des Minimums und gibt, wie eine triviale Rechnung zeigt, den Abstand von der Minimalebene an, in dem die Intensität den doppelten Wert der im Minimum vorhandenen Intensität hat. $2 \mathrm{Im} I$ ist in diesem Sinne also die Doppelwertsbreite des Minimums der reflektierten Welle.

Die Ergebnisse von II gelten unter der einzigen einschränkenden Voraussetzung: $\Delta \ll 1$, die bei allen in dieser Arbeit interessierenden Fällen erfüllt ist. Die völlig allgemeine und strenge Lösung

$$
\mathfrak{D}=\frac{i}{\pi n_{1} \sqrt{2(1-\cos \Delta)}} \operatorname{arctg}\left(\begin{array}{l}
1 \mathrm{r}-\mathfrak{r}^{\prime} \\
i \mathrm{r}+\mathbf{r}^{\prime}
\end{array}\right)
$$

sei hier nur erwähnt; sie folgt auch leicht aus Gl. (6).
III. Untersuchung verschiedener Spezialfälle mit vereinfachter Theorie

Zur Gewinnung eines ersten Überblicks soll hier die Gl. (7) durch den Grenzübergang $\Delta \rightarrow 0$ besonders vereinfacht werden. Man erhält dann

$$
\left.\mathfrak{D}=\frac{\lambda}{2 \pi i n_{1}} \frac{1}{\mathfrak{r}} d \varphi_{1} \quad \text { für }\right\lrcorner \rightarrow 0 .
$$

Zum Beispiel liest man aus Gl. (12) ganz unmittelbar $\mathrm{ab}$, daß $\mathfrak{I}$ rein imaginär ist, wenn $\mathfrak{r}$ reell ist, wenn also weder Totalreflexion noch Absorption hineinspielen. Dann findet nur eine Verschmierung des Minimums, aber keine Versetzung statt. Gl. (12) kann man auch umschreiben:

$$
\mathfrak{D}=\frac{i}{2 \pi n_{1}} \frac{d}{d \varphi_{1}} \operatorname{arc} \mathfrak{r}-i \frac{\lambda}{2 \pi \mathrm{n}_{1}} \frac{1}{\mathrm{r}} \frac{d}{d \varphi_{1}} \mathrm{r} .
$$

An dieser Form erkennt man unmittelbar, daß z. B. bei Totalreflexion (hier ist ja $|\mathfrak{r}|=1$ ) das Minimum einwandfrei scharf bleibt und eine Versetzung je nach der Abhängigkeit des arc $\mathfrak{r}$ vom Einfallswinkel erfährt.

Allgemein ist eine solche Änderung des arc $\mathfrak{r}$ mit dem Einfallswinkel ersichtlich die notwendige und hinreichende Bedingung für das Auftreten einer Versetzung. Die für (12) erforderliche logarithmische Ableitung des Reflexionskoeffizienten ist leicht zu berechnen und führt naturgemäß zu verschiedenen Werten je nach Polarisation.

$$
\mathfrak{D}_{\mathrm{s}}=\frac{i}{i \pi} \frac{\sin \varphi_{1}}{\mathrm{n}_{2} \cos \varphi_{2}} ; \quad \mathfrak{D}_{\mathrm{p}}=\mathfrak{D}_{\mathrm{s}} \frac{1}{\sin ^{2} \varphi_{1}\left(1+n_{1}{ }^{2} / \mathfrak{n}_{2}{ }^{2}\right)-1}
$$

Diese Gleichungen benutzen wir zur Untersuchung einiger spezieller Fälle, beschränken uns jedoch auf die im Rahmen unserer Zielsetzung wichtigen.

1. Spezialfall: Beide Medien ohne Absorption, keine Totalreflexion; dann tritt keine Versetzung ein, aber eine Minimumverschmierung des Betrages

$$
\begin{gathered}
\operatorname{Im} \mathfrak{D}_{\mathrm{s}}=\frac{\lambda}{\pi} \frac{\sin \varphi_{1}}{\mathfrak{n}_{2} \cos \varphi_{2}} \\
\operatorname{Im} \mathfrak{D}_{\mathrm{p}}=\operatorname{Im} \mathfrak{D}_{\mathrm{s}} \frac{1}{\sin ^{2} \varphi_{1}\left(1+n_{1}{ }^{2} / \mathfrak{n}_{2}{ }^{2}\right)-1} .
\end{gathered}
$$

Bei einer unter $45^{\circ}$ von Luft auf Glas auffallenden Welle gibt es also eine Minimumverschmierung um 0,213 Wellenlängen bei senkrechter und 0,775 Wellenlängen bei paralleler Polarisation.

Wir wollen nun diesen Fall ausschließen und also $\mathrm{n}_{2} \cos \varphi_{2}$ als komplex ansehen; die einfallende Welle sei homogen, $\sin \varphi_{1}$ reell. Dann steht die erste der Gleichungen (15) ersichtlich in enger Beziehung zu der bekannten Formel für die Eindringtiefe $F$ der 
Welle in das Medium 2. Darunter werde die Tiefe verstanden, in der die eindringende Welle noch den e-ten Teil der Amplitude besitzt, die an der Grenzfläche selbst vorlag. Die Feldstärke der eindringenden Welle ist nach Abb. 1

$$
\begin{aligned}
& E_{\mathrm{d}}(\mathrm{x}, \mathrm{y}, \mathrm{z}) \\
& =E_{\mathrm{d}}(0,0,0) \exp \left[-i \frac{2 . \pi}{i} \mathrm{n}_{2}\left(x \sin \varphi_{2}-z \cos \varphi_{2}\right)\right] .
\end{aligned}
$$

Wegen des Brechungsgesetzes folgt:

$$
\begin{aligned}
& E_{\mathrm{d}}(\mathrm{x}, \mathrm{y}, \mathrm{z})=E_{\mathrm{d}}(0,0,0) \exp \left[-i^{2 . \top} n_{1} x \sin \varphi_{1}\right] \\
& \cdot \exp \left[\begin{array}{cc}
2 . \pi \mathrm{n}_{2} & \approx \cos \varphi_{2} \\
i &
\end{array}\right], \text { d.h. } \\
& E_{\mathrm{d}}(\mathrm{x}, \mathrm{y}, \mathrm{z})=E_{\mathrm{d}}(0,0,0) \exp (z / F) ; \\
& F=\operatorname{Re}\left(\begin{array}{cc}
i & 1 \\
i 2 . \tau & \mathrm{n}_{2} \cos \phi_{2}
\end{array}\right) .
\end{aligned}
$$

Nach (12) und (15) ist also

$$
D_{\mathrm{s}}=\operatorname{Re} \mathfrak{D}_{\mathrm{s}}=2 F \sin \varphi_{1} .
$$

Eine besonders anschauliche Form nimmt diese Beziehung an, wenn wir die Tiefe der scheinbaren Reflexionsebene berechnen; diese sei mit $Z$ bezeichnet; nach Abb. 1 ist $D=Z 2 \sin \varphi_{1}$. Allgemein definieren wir eine entsprechende komplexe Größe mit $3=I / 2 \sin \varphi_{1}$. Offenbar ist dann

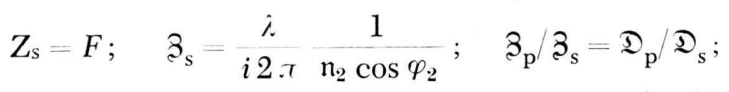

für senkrechte Polarisation ist also die Tiefe der scheinbaren Reflexionsebene gleich der Eindringtiefe; für parallele Polarisation sind die Verhältnisse etwas komplizierter, aber aus (15) ohne weiteres zu entnehmen. Das mag in Spezialfällen hier ausgeführt werden.

2. Spezialfall: Medium 1 sei Luft, Medium 2 Seewasser der spezif. Leitfähigkeit $\sigma=10^{-2} \Omega^{-1} \mathrm{~cm}^{-1}=9 \cdot 10^{9}$ el.st. CGS. Die Wellenlänge betrage etwa $10 \mathrm{~km}$. Der Brechungsindex des Seewassers ist dann nach der Drudeschen Beziehung

$$
\mathfrak{n}_{2}=\sqrt{\varepsilon-i}{ }_{c}^{2 \sigma i}=(1-i) \sqrt{\frac{2 \sigma i}{c}}=(1-i) 775,
$$

da die Dielektrizitätskonstante $\varepsilon=80 \ll \frac{2 \sigma i}{\mathrm{c}}=6 \cdot 10^{5}$ ist und chenso vernachlässigt werden kann wie das $\sin ^{2} \varphi_{1}$ in

4 Landolt-Börnstein, Physikalisch-Chemische Tabellen, 1. Ergänzungsband, S. 463; Springer 1927. der folgenden Berechnung des Nenners von Gl. (18)

$$
\mathrm{n}_{2} \cos \varphi_{2}=\sqrt{\mathrm{n}_{2}{ }^{2}-\sin ^{2} \varphi_{1}}=\left|\mathfrak{n}_{2}{ }^{2}=(1-i)\right|_{c}^{2 \sigma i} .
$$

Somit ergibt sich die Eindringtiefe nach Gl. (19) zu

$$
F=\frac{1}{4 . \tau} \sqrt{\frac{c i}{2 \sigma}}=Z_{\mathrm{s}}=98 \mathrm{~cm} .
$$

Für parallele Polarisation erhält man im Nenner der zweiten Gl. (15) nahezu $\sin ^{2} \varphi_{1}-1$, da das Reziproke von $\mathrm{It}_{2} 2$ eine dem Betrage nach sehr kleine Zahl ist; daher wird

$$
Z_{\mathrm{p}}=-\frac{F}{\cos ^{2} \varphi_{1}} .
$$

Diese Strahlversetzung ist also dem Betrage nach größer als bei senkrechter Polarisation; ihr Vorzeichen bedeutet offenbar, daß die scheinbare Reflexionsebene oberhalb der Wasseroberfläche liegt. Eine ähnliche, paradox erscheinende Beobachtung an Längstwellen (freilich im Nahfeld der Sendeantenne) veranlaßte 1940 den Verf. zu den hier mitgeteilten Rechnungen und letzten Endes auch zur Übertragung der Minimumstrahlkennzeichnung auf die Optik. Trotz der Größe der Versetzung ist eine Messung sehr schwierig, da die Doppelwertsbreite des Minimums in derselben Größe liegt.

3. Spezialfall: Medium 1 sei nichtleitend. Medium 2 sei Metall, z. B. Silber. $Z_{\mathrm{s}}$ ist jedenfalls gleich der Eindringtiefe nach Gl. (20), also wesentlich kleiner als die Dicke einer undurchsichtigen Schicht des Metalls. Nach der oben gegebenen Definition von 3 und Gl. (20) ist zunächst

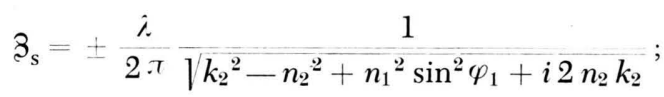

darin ist $\pi_{2}=n_{2}-i k_{2}$, wie üblich, gesetzt worden. Die Vorzeichenbestimmung in Gl. (23) hat so zu geschehen, daß $F=\operatorname{Re} 3$ s positiv ausfällt; das ist erforderlich, damit der Exponent im Abklingfaktor der ersten Gl. (18) für negative $z$ negativ wird, wie es das Energieprinzip erzwingt.

Nach M in or 4 ist im Sichtbaren bei Silber weder der Brechungsindex noch der Absorptionskoeffizient stark von der Wellenlänge abhängig; wir erhalten daher einen für das ganze sichtbare Gebiet annähernd gültigen Überblick, wenn wir z. B. die Werte für $\lambda=5500 \AA\left(k_{2}=3,31\right.$; $\left.n_{2}=0,18\right)$ einsetzen. Dann wird bei einem Einfallswinkel $\varphi_{1}=45^{\circ}$

$$
\S_{\mathrm{s}}=i 0,046(1-i 0,05) ; \quad Z_{\mathrm{s}}=2,6 \cdot 10^{-6} \mathrm{~cm} .
$$

Der sehr kleine Imaginärteil zeigt, wie scharf das Minimum auch nach der Reflexion noch ist; seine Doppelwertsbreite beträgt nur $10 \%$ der an sich schon sehr kleinen Strahlversetzung bei dem Silber.

Nach der Definition von 3 und der zweiten Gl. (14) berechnet man leicht

$$
3_{\mathrm{p}}=3_{\mathrm{s}}(-1,84-i 0,02)=-4,6 \cdot 10^{-6} \mathrm{~cm} .
$$

Die Strahlversetzung für parallele Polarisation ist bei der Reflexion an dicken Silberschichten im Sichtbaren 
negativ, d. h. der Strahl wird reflektiert, als ob die Reflexion vor der Oberfläche geschehen wäre; bei senkrechter Polarisation ist sie, wie stets, positiv. In beiden Fällen ist sie klein und liegt unter $10 \%$ der Wellenlänge.

4. Spezialfall: Medium 1 und 2 seien durchsichtig, 1 sei das dichtere Medium $\left(n_{1}>n_{2}\right)$. Der Einfallswinkel sei größer als der Grenzwinkel der Totalreflexion $\varphi_{g} ;$ d. h. $\sin \varphi_{1}>\sin \varphi_{\mathrm{g}}=n_{\mathrm{g}} / n_{1}$. Der einfallende Strahl wird dann total reflektiert, und wir haben den von Goos und Hänchen experimentell und von Artmann theoretisch behandelten Fall vor uns, dessen Untersuchung mit der Methode der Minimumstrahlkennzeichnung Hauptgegenstand dieser Arbeit ist. Nach Gl. (20) wird

$$
\begin{aligned}
& 3_{\mathrm{s}}=\stackrel{i}{2 . \tau} i_{i}^{n_{2} \cos \varphi_{2}}=\frac{i}{2 . \top i \sqrt{n_{2}{ }^{2}-n_{1}{ }^{2} \sin ^{2} \varphi_{1}}} \\
& i \\
& 2 . \tau \sqrt{n_{1}{ }^{2} \sin ^{2} \psi_{1}-n_{2}{ }^{2}} \\
& 3_{\mathrm{s}}=F=\frac{i}{2 . \tau n_{1} \sqrt{\sin ^{2} \varphi_{1}}-\sin ^{2} \varphi_{\mathrm{g}}} \\
& \lambda \\
& 2.7 n_{1} / \sin \left(\varphi_{1}+\varphi_{\mathrm{g}}\right) \sqrt{\sin \left(\varphi_{1}-\varphi_{\mathrm{g}}\right)} .
\end{aligned}
$$

Da 3 ; also rein reell ist, ist das Minimum auch nach der Totalreflexion völlig scharf; es ist so parallel verschoben, daß die Reflexion nicht an der Grenzfläche, sondern im dünneren Medium geschehen zu sein scheint - und zwar in einer Tiefe gleich der Eindringtiefe der ins dünnere Medium eindringenden inhomogenen Welle.

Diese Tiefe ist nach Gl. (27) offenbar für großen Abstand des Einfallswinkels vom Grenzwinkel nur wenig größer als $\lambda / 2 \pi n_{1}$, d.h. für Glas-Luft nur wenig größer als $10 \%$ der Wellenlänge. Nähert sich der Einfallswinkel dem Grenzwinkel, so wächst die Tiefe unbegrenzt, und zwar nahe am Grenzwinkel umgekehrt proportional mit $\rceil \varphi_{1}-\varphi_{\mathrm{g}}$. Abb. 2 zeigt mit ihrer unteren Kurve diesen Verlauf für senkrechte Polarisation bei einer Glas-Luft-Grenze mit $n_{1}=1,52$ und $n_{2}=1$.

Die obere der beiden Kurven gibt das gleiche für parallele Polarisation; man erhält sie aus den Gln. (14), (20) und (27); es ist hier 3p reell, und zwar

$$
Z_{\mathrm{p}}=F_{\sin ^{2} \varphi_{1}\left(1+{n_{1}}^{2} / n_{2}{ }^{2}\right)-1}
$$

der Faktor bei $F$ hat nahe am Grenzwinkel offenbar den Wert $n_{1}{ }^{2} / n_{2}{ }^{2}$ - das ist in unserem Beispiel 2,3und wird für wachsenden Einfallswinkel $\varphi_{1}$ langsam kleiner.
Diese Ergebnisse stimmen mit denen Artmanns, die für einen Maximumstrahl gewonnen waren, überein. Man berücksichtige, daß die Formeln von Artmann nur sehr nahe am Grenzwinkel der Totalreflexion gelten. Theoretisch führen also die Maximummethode und die Minimummethode (in unserer bisherigen einfachen und - wie wir noch sehen werden - allerdings nicht ausreichenden Form) zu dem gleichen Ergebnis. Formal sind besonders die Eingangsbetrachtungen von Artmann einem Teil der hier mitgeteilten auch durchaus ähnlich trotz der unterschiedlichen Zielsetzung. Damit ist wahrscheinlich geworden, daj der Zwiespalt zwischen den Rechnungen von Art-

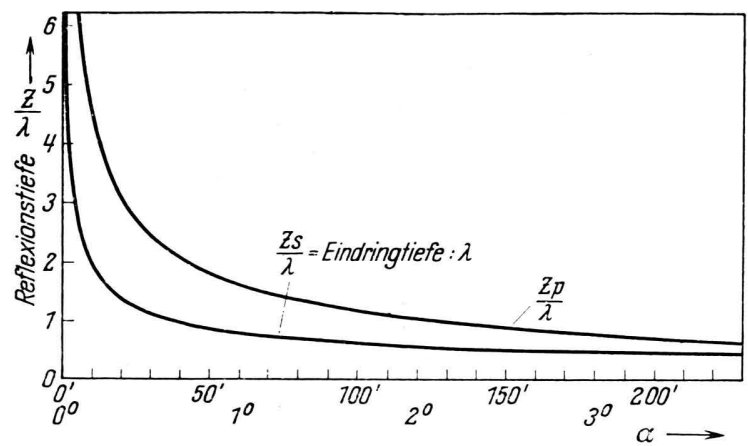

Abb. 2. Scheinbare Reflexionstiefe $Z$ bei der Totalreflexion an einer Glas-Luft-Grenze als Funktion der Differenz $\alpha$ des Einfallswinkels vom Grenzwinkel der Totalreflexion, berechnet nach der vereinfachten Theorie für $\Delta \rightarrow 0$.

mann und den experimentellen Ergebnissen von Goos und Hänchen nicht durch die von Artmann benutzte Kirchhoffsche Formel, sondern durch die Subtilität des Effekts und die außerordentlichen experimentellen Schwierigkeiten, ihn hinreichend genau zu messen, bedingt ist. Die Meßgenauigkeit ist bei Goos und Hänchen durch die prinzipielle Strahlunschärfe beschränkt; die Minimumstrahlkennzeichnung setzt uns in die Lage, die Strahlunschärfe als das die Meßgenauigkeit begrenzende Element auszuschalten und also die Messungen so weit zu treiben, wie es die Mängel der zur Verfügung stehenden optischen Hilfsmittel, insbesondere der total reflektierenden Grenzfläche selbst gestatten.

IV. Meßapparatur

und erste Beobachtungsergebnisse

Die Apparatur muß vor allem die von der Theorie vorausgesetzten Bedingungen realisieren. Die Minima, deren Versetzung wir messen wollen, müssen daher durch Interferenz zweier ebener Wellen entstehen, 
und wir verwenden daher das Verdoppelungsveriahren im Sinne der vorhergehenden Arbeit, hier speziell mit kleinen Änderungen das klassische Biprismenverfahren von Fresnel.

Abb. 3 zeigt eine Skizze der Apparatur. Ein Glühlampenfaden $\mathrm{L}$ wurde mittels zweier Kondensorlinsen $\mathrm{K}_{1}, \mathrm{~K}_{2}$ über Monochromatfilter $\mathrm{F}$ und das Polaroidfilter $\mathrm{P}$ auf den Spalt $\mathrm{Sp}$ abgebildet, der so zu einer fadenförmigen Lichtquelle fast monochromatischen, polarisierten Lichtes wurde. Ein reell abbildendes Biprisma Bp und die Linse Li machten daraus zwei reelle Spaltbilder D, die als kohärente, gleichstarke Lichtquellen von 1,06 mm Abstand dienten und deren Phasenbeziehung mittels des -
Die in dieser Objektebene vorhandene Lichtverteilung wurde rund 4-mal vergrößert auf den Film Fi (meist Isopan F) abgebildet, um die Größe des Silberkorns nicht allzusehr zur Auswirkung kommen zu lassen. Eine rund 7 -fache Nachvergrößerung des Filmes führte dann zu Aufnahmen, wie Abb. 4 sie zeigt (s. Tafel S. 144 a).

Freilich sind in dieser Aufnahme nur das obere und das untere Drittel so behandelt worden wie beschrieben. Das mittlere Drittel des Lichtes wurde an der Rückseite der Planparallelplatte nicht total, sondern zur Schaffung eines Vergleichstrahles an einem Streifen Silber reflektiert, der sich horizontal von der linken bis zur rechten Kante der Planparallelplatte erstreckte. Die Planparallelplatte war zu diesem Zwecke wie bei Goos und Hänchen hergerich-

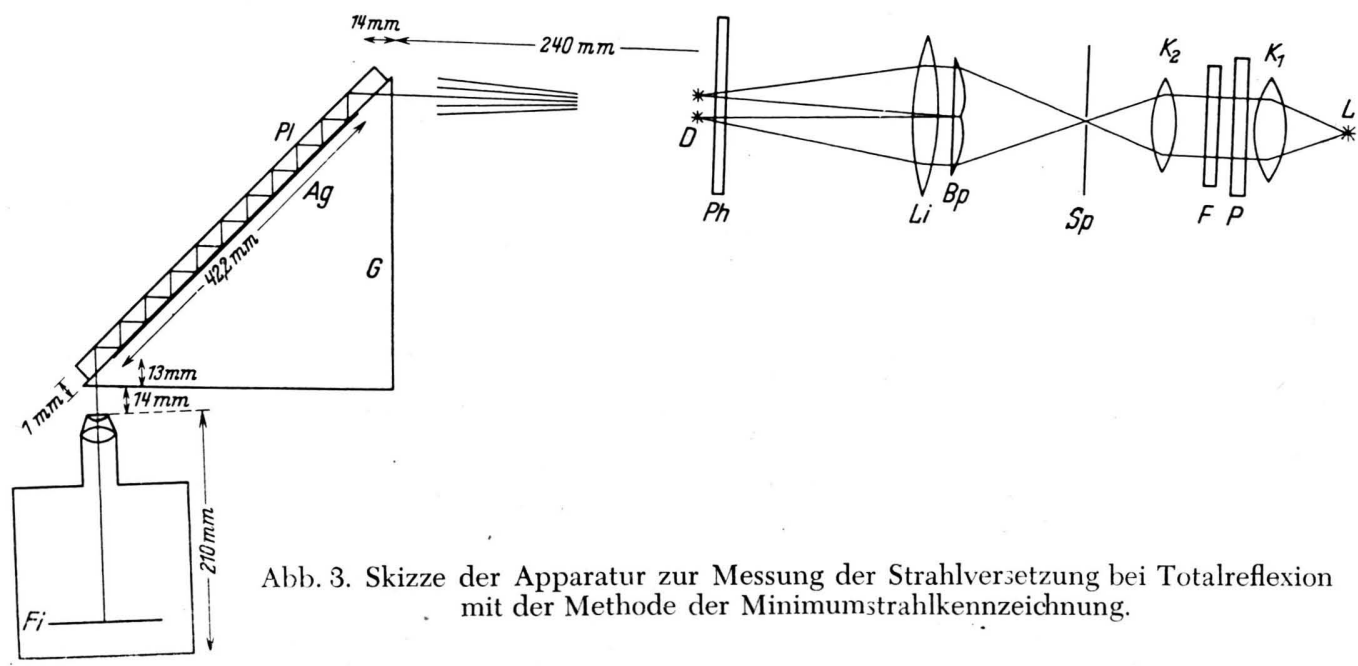

übrigens nur für visuelle Beobachtungen eingesetzten und hier nicht weiter zu diskutierenden - Phasenschiebers Ph verändert werden konnte.

Bei den ersten Messungen war noch eine Sammellinse (in der Abb. 3 nicht gezeichnet) so eingesetzt worden, daß die Nullebenen parallel zueinander lagen und durch Interferenz zweier ebener Wellen in einer unserer Theorie völlig entsprechenden Weise entstanden. Doch erwies sich die Linse als überflüssig, da die Wellen in dem kleinen Gebiet, in dem sie gebraucht wurden, ohnehin ausreichend eben waren. Zugleich war aber die leichte Änderung des Einfallswinkels von einer Nullebene zur anderen ein Vorteil, da mehrere Einfallswinkel zugleich auf einer Aufnahme erfaßt werden konnten.

Das so mit leicht divergenten Nullebenen gekennzeichnete Licht wurde durch ein Glasprisma $G$ in eine mit Immersionsöl aufgekittete $1 \mathrm{~mm}$ dicke Planparallelplatte Pl geschickt, an deren Rückseite es zum ersten Male totalreflektiert wurde. An einer zwischen Prisma und Platt? liegenden Ag-Schicht Ag wurde es erneut reflektiert und so insgesamt $25+24$-mal auf seinem Zickzackwege reflektiert, und zwar 25-mal total an der Glas-Luft-Grenze und 24-mal an der Ag-Fläche. (In die Abb. 3 sind der Übersichtlichkeit wegen wesentlich weniger Reflexionen (ingezeichnet.) Nachdem das Licht schließlich wieder das Prisma passiert hatte, trat es in die Mikrokamera ein, deren Objektebene E noch innerhalb des Glasprismas lag. tet. Die Silberschicht an der Vorderseite der Planparallelplatte und der genannte Silberstreifen waren im Hochvakuum aufgedampft.

Abb. 4 p zeigt, wie der totalreflektierte Strahl (genau genommen jede Nullebene) gegenüber dem an der Silberschicht reflektierten versetzt wird. Doch ist nur der rechte - etwa 5 Minima enthaltende Teil der Aufnahme zur Auswertung heranzuziehen, da der linke nur 24-mal totalreflektiert wurde; die Breite der vorderen Silberfläche hatte zur nochmaligen Reflexion nicht ganz ausgereicht. Die entsprechende Erscheinung behandeln G. u. H. ${ }^{1}$ ausführlicher. Da der Silberstreifen auf der Rückseite der Planparallelplatte an seinem einen Ende eine etwas größere Breite hatte als auf dem anderen, ist der Übergang zwischen den in der Aufnahme abgebildeten Minima so langsam abgestuft, daß bezüglich der Zuordnung kein Zweifel entstehen kann. Betrachtet man die Aufnahme schräg von unten in der Richtung der Minima, so erkennt man, daß z. B. bei dem 4. Minimum (von rechts aus gezählt) die Versetzung etwa gleich einem Streifenabstand ist. 
Das galt für parallele Polarisation; Abb. $4 \mathrm{~s}$ gibt dasselbe für senkrechte Polarisation unter sonst völlig gleichen Bedingungen. Der vierte Streifen von rechts zeigt nur eine Versetzung um nicht ganz einen halben Streifenabstand; das Polarisationsverhältnis $D_{\mathrm{l}} / D_{\mathrm{s}}$ hat den Wert 2,3 , wie die Theorie es verlangt hatte.

Zeigte sich so schon bald nach Beginn der experimentellen Untersuchungen eine volle Übereinstimmung bezüglich des Polarisationsverhältnisses zwischen Theorie und Experiment, so ergab die absolute Messung der Verschiebung selbst sofort Abweichungen von der Theorie, die weit außerhalb der Meßfehlergrenzen lagen.

Der Grund hierfür liegt in der Vorzussetzung $\Delta \rightarrow 0$ der bisherigen Theorie. Diese Voraussetzung ist im Experiment zwar insofern erfüllt, als $\Delta \ll 1$ ist; aber $\Delta$ ist nicht klein gegen den Abstand der Einfallswinkel vom Grenzwinkel der Totalreflexion. Um der Theorie auch hierin zu entsprechen, müßte man entweder bei gleichem $\Delta$ die Einfallswinkel vergrößern oder andernfalls $\Delta$ sehr stark verkleinern. Beides führt zu einer unmeßbaren Strahlversetzung; diese nimmt selbst im ersten Fall stark ab, wie Abb. 2 zeigt. Für abnehmendes $\Delta$ bei gleichem Einfallswinkel wächst allerdings die Strahlversetzung, aber in noch höherem Maße auch die Strahlunschärfe. Die prinzipiellen Hintergründe hierfür erkennt man am klarsten unter Verwendung des in der vorhergehenden Arbeit eingeführten Begriffs der Subtilität.

Unter der Subtilität eines Effekts ist die Größe

\section{Wellenlänge \\ Koordinatendifferenz $\times$ Winkeldifferenz

zu verstehen, diese ist nach Gl. (7) umgekehrt proportional zu $\mathrm{r}-\mathrm{r}^{\prime}$, wächst also unbeschränkt, wenn $\Delta \rightarrow 0$ geht. Das bedeutet aber, daß an die Strahlschärfe, die nach dem in der vorhergehenden Arbeit ausgesprechenen Satz mindestens gleich der Subtilität des zu messenden Effektes sein muß, ebenfalls unbeschränkt hohe Anforderungen zu stellen wären. Diese Dinge werden in VIII noch quantitativ betrachtet werden; es ist jedoch hier bereits zu erkennen, daß eine Verbesserung der Theorie unter Verzicht auf den Grenzübergang $\Delta \rightarrow 0$ unumgänglich ist; denn dieser Grenzfall selbst muß sich wegen Divergenz der Subtilität jedenfalls der Beobachtung entziehen, und eine weitere Annäherung an ihn muß die Schwierigkeiten nur erhöhen.

\section{Verbesserte Theorie}

Die Voraussetzung $A \rightarrow 0$ wurde erst zu Beginn des Abschnitts III gemacht. Da wir sie nunmehr fallen lassen, haben wir auf die Ergebnisse von II zurückzugreifen, die unter der praktisch stets zutreffenden Voraussetzung $A \ll 1$ galten. Damit entfällt insbesondere jede Einschränkung bezüglich der Größenver- hältnisse zwischen dem Kreuzungswinkel und den Abständen der Einfallswinkel vom Grenzwinkel der Totalreflexion. Wir haben in Gleichung (7) die Werte für die Fresnelschen Reflexionskoeffizienten einzusetzen und erhalten nach einfacher Zwischenrechnung

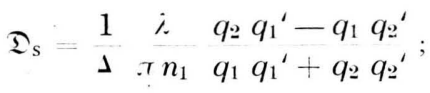

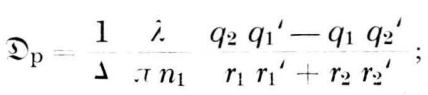

darin bedeuten die $q$ und $r$ bei Totalreflexion reelle Zahlen

$$
\begin{gathered}
q_{1}=n_{1} \cos \varphi_{1} ; \quad q_{2}=i n_{2} \cos \varphi_{2} ; \quad r_{1}=n_{2} \cos \varphi_{1} ; \\
r_{2}=i n_{1} \cos \varphi_{2} ; \\
q_{1}{ }^{\prime}=n_{1} \cos \varphi_{1}^{\prime} ; \quad q_{2}{ }^{\prime}=i n_{2} \cos \varphi_{2}^{\prime} ; \quad r_{1}{ }^{\prime}=n_{2} \cos \varphi_{1}^{\prime} ; \\
r_{2}{ }^{\prime}=i n_{1} \cos \varphi_{2}{ }^{\prime} .
\end{gathered}
$$

Für quantitative Betrachtungen werden wir diese Formeln heranziehen. Doch läßt sich für qualitative Untersuchungen die Gl. (30) wesentlich einfacher schreiben, wenn wir nahe am Grenzwinkel der Totalreflexion beobachten, genauer: wenn von den Beziehungen

$$
u=\varphi_{1}-\varphi_{\mathrm{g}} \ll 1 ; \quad \iota^{\prime}=\varphi^{\prime}{ }_{1}-\varphi_{\mathrm{g}} \ll 1
$$

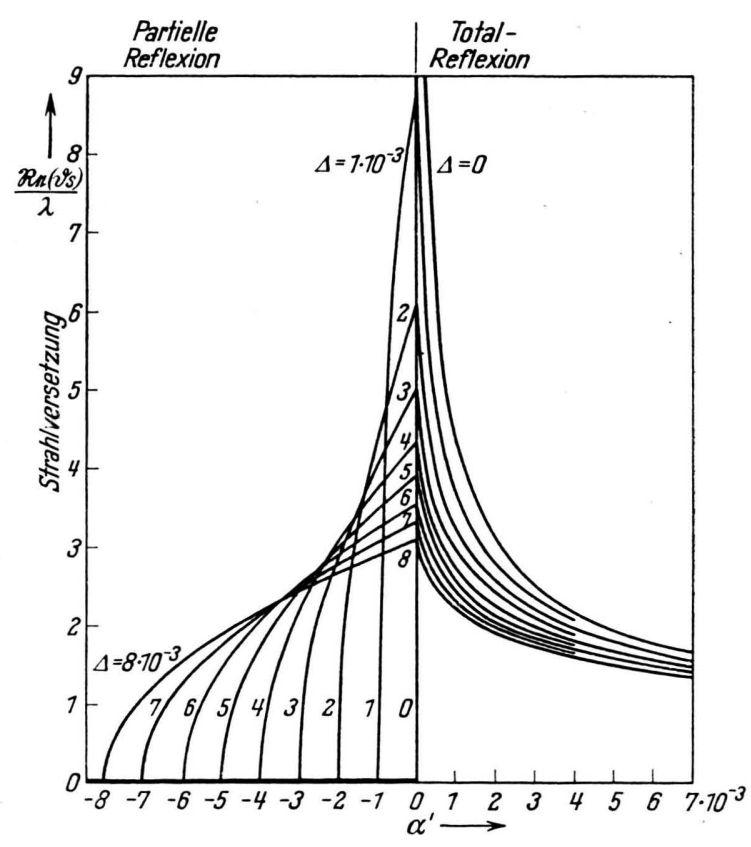

Abb. 5. Nach der verbesserten Theorie berechnete Strahlversetzung $D$ als Funktion der Differenz $\alpha^{\prime}$ des Einfallswinkels vom Grenzwinkel der Totalreflexion. 
mindestens eine erfüllt ist; denn dann gilt in guter Näherung

$$
\stackrel{\mathfrak{D}_{\mathrm{s}}}{i}=\underset{\tau n_{1} \mid n_{1}^{2} / n_{2}{ }^{2}-1}{12} \mid \iota^{\prime}+\Delta-l^{\prime \prime} ;
$$

darin ist $a^{\prime}=\varphi_{1}^{\prime}-\varphi_{\underline{m}}$ der Abstand der ,steileren“ Welle vom Grenzwinkel der Totalreflexion.
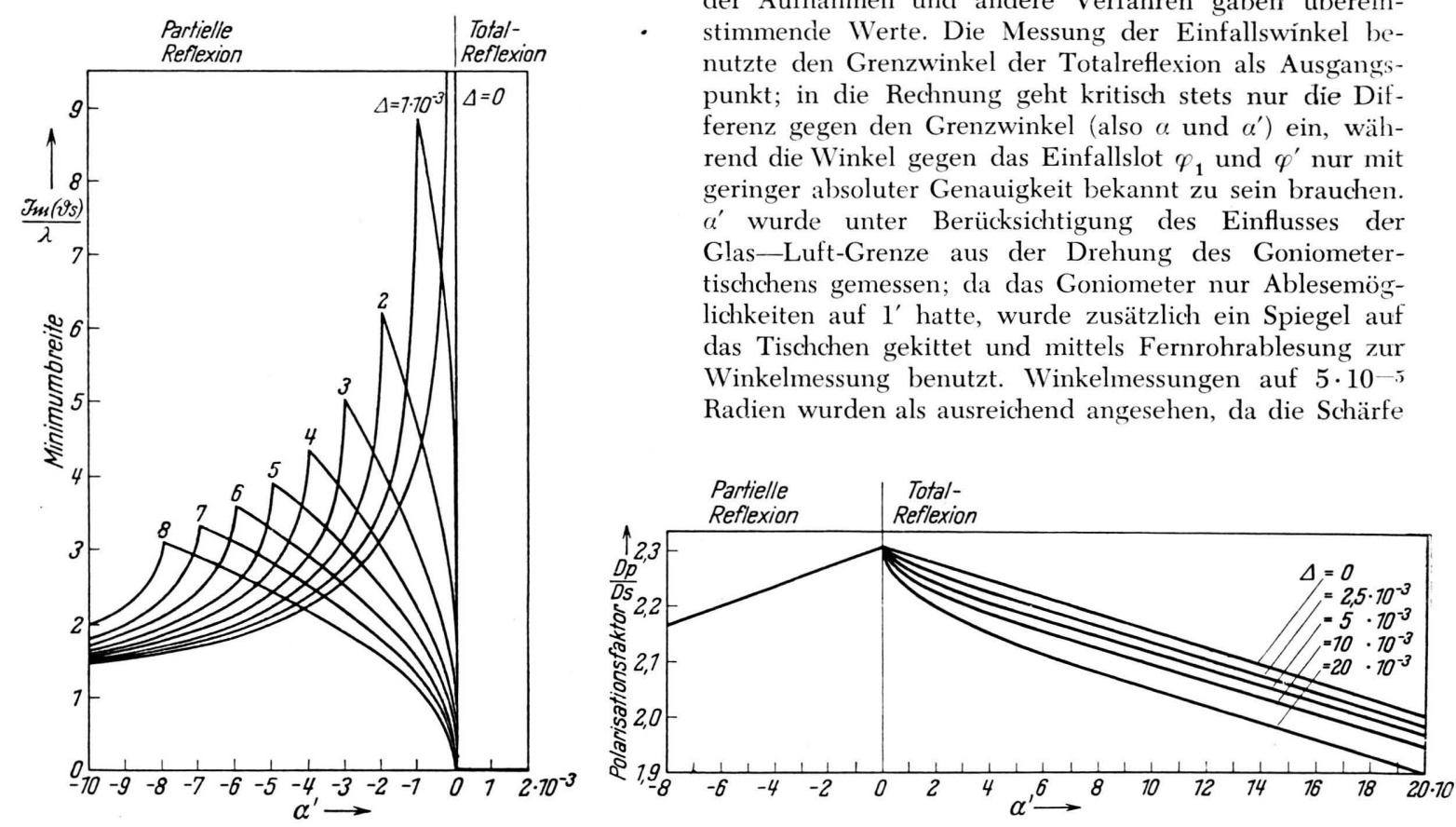

Abb. 6. Nach der verbesserten Theorie berechnete Minimumverschmierung als Funktion der Differenz $\alpha^{\prime}$ des Einfallswinkels vom Grenzwinkel der Totalreflexion.

Abb. 5 zeigt den Verlauf des Realteils, also der Strahlversetzung, und Abb. 6 den des Imaginärteils, also der Minimumverschmierung. Die in Abb. 5 für $\Delta=0$ gezeichnete Kurve ist gleichbedeutend mit der unteren der Kurven in Abb. 2, jedoch gibt sie im Gegensatz zu jẹner mit Rücksicht auf den Vergleich mit den Messungen bereits die unmittelbar experimentell zugängliche Versetzung statt der scheinbaren Tiefe der Reflexionsebene, die in unserer strengeren Theorie doch keine einfache anschauliche Beziehung zur Eindringtiefe befolgt. Abb. 7 gibt das Polarisationsverhältnis.

VI. Vergleich der Messungen mit der verbesserten Theorie

Die Messungen stützten sich auf visuelle Beobachtungen ${ }^{5}$ und photographische Registrierungen, in

5 Diese hatten mehr orientierenden Charakter und bestätigten vor allem die Abhängigkeit der Versetzung vom Kreuzungswinkel in der durch Abb. 5 beschriebenen Weise. erster Linie auf 26 Filme mit durchschnittlich je 7 Aufnahmen und insgesamt etwas mehr als 300 auswertbaren Minima.

Der Kreuzungswinkel wurde bei den Aufnahmen auf 2,5 $10^{-3}$ Radien eingestellt. Er wurde am genauesten aus der geometrischen Anordnung (unter Berücksichtigung der modifizierenden Wirkung der Glas-Luft-Grenze) gemessen; Berechnung aus den Interferenzstreifenabständen der Aufnahmen und andere Verfahren gaben übereinstimmende Werte. Die Messung der Einfallswinkel benutzte den Grenzwinkel der Totalreflexion als Ausgangspunkt; in die Rechnung geht kritisch stets nur die Differenz gegen den Grenzwinkel (also $\alpha$ und $\alpha^{\prime}$ ) ein, während die Winkel gegen das Einfallslot $\varphi_{1}$ und $\varphi^{\prime}$ nur mit geringer absoluter Genauigkeit bekannt zu sein brauchen. $a^{\prime}$ wurde unter Berücksichtigung des Einflusses der Glas-Luft-Grenze aus der Drehung des Goniometertischchens gemessen; da das Goniometer nur Ablesemöglichkeiten auf $1^{\prime}$ hatte, wurde zusätzlich ein Spiegel auf das Tischchen gekittet und mittels Fernrohrablesung zur Winkelmessung benutzt. Winkelmessungen auf $5 \cdot 10^{-5}$ Radien wurden als ausreichend angesehen, da die Schärfe

Abb. 7. Nach der verbesserten Theorie berechnetes Verhältnis der Strahlversetzung beider Polarisationen als Funktion der Differenz $\alpha^{\prime}$ des Einfallswinkels vom Grenzwinkel der Totalreflexion.

der Totalreflexionsgrenze selbst bei Verwendung der Filterkombination RG1 + BG 18 (Schott), die nur einen schmalen Bereich im Roten mit Schwerpunkt bei 6180 I durchließ, nur auf etwa $20 \cdot 10^{-5}$ Radien definiert schien. Gelegentlich wurde auch die grüne Hg-Linie benutzt, ohne daß die Genauigkeit dadurch merklich erhöht worden wäre. Innerhalb einer Aufnahme ändert sich der Einfallswinkel von Minimum zu Minimum, wie eine elementare Rechnung zeigt, um den Winkel $\lambda / g$ 1,52, wenn $g$ den Abstand der beiden reellen Spaltbilder (in unserem Falle meist $1,06 \mathrm{~mm}$ ) bezeichnet.

Die Minimumverschiebung wurde vorwiegend an den Negativen selbst mit einem Komparator (Ablesung auf $1 / 1000 \mathrm{~mm}$ ) gemessen. Ist $\xi$ das Ergebnis dieser Messung, so berechnet sich daraus die Strahlversetzung $D$ durch Reduktion um die Vergrößerung der Mikrokamera $V_{1}$ und die Vervielfachung (Zahl der wirksamen Totalreflexionen $N$ ) gemäß

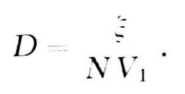


An den vergrößerten Positiven, z. B. den Abb. 4, kann die Messung unmittelbar mit einem Maßstab geschehen. Da dort $N=25, \quad V_{1}=4$ und die Nachvergrößerung $V_{\underline{2}}=7$ ist, gilt hier $D=\xi / 700$.

Hieran ist wegen der Versetzung am Silber eine Korrektur anzubringen; bei $D_{\mathrm{s}} / \lambda$ ist $0,067 \mathrm{zu}$ addieren, bei $D_{p} / \lambda$ wurde 0,118 subtrahiert. (Ohne sie erschiene das Polarisationsverhältnis noch etwas ausgeprägter.)

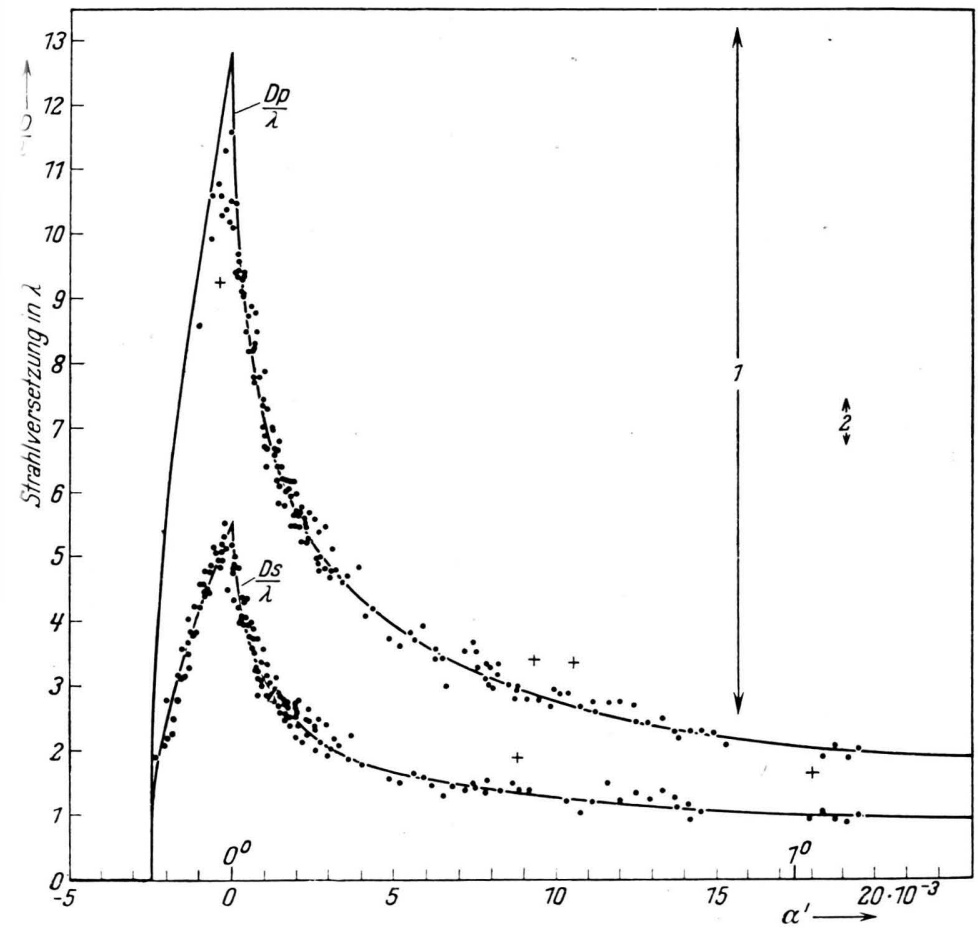

Abb. 8. Gemessene Strahlversetzungen (Punkte) bei einem Kreuzungswinkel von $2,5 \cdot 10^{-3}$. Radien im Vergleich zur verbesserten Theorie (ausgezogene Kurven). Die Abszisse gibt die Differenz $\alpha^{\prime}$ des Einfallswinkels vom Grenzwinkel der Totalreflexion. $(\leftarrow 1 \rightarrow$ entspricht der Mindestbreite des Lichtbandes bei Goos u. Hänchen; $\leftarrow 2 \rightarrow$ entspricht der Doppelwertsbreite eines Minimums.)

Das Ergebnis dieser Auswertung wurde durch die Meßpunkte in Abb. 8 dargestellt; zum Vergleich sind die nach Gl. (30) bzw. (32) für $2,5 \cdot 10^{-3}$ Radien berechneten theoretischen Kurven eingetragen. Die Polarisationsabhängigkeit liegt weit außerhalb der Streuung und kann als experimentell gesichert gelten. Die rechts in Abb. 8 veranschaulichte Breite des Lichtbandes bei den Messungen von Goos und Hänchen und die dort angegebene Minimumbreite sind bereits um die Vervielfachung ( 34 bzw. 25 ) so weit reduziert, daß sie unmittelbar mit den Werten von $D / \lambda$ zur Beurteilung der Meßgenauigkeit verglichen werden können. Hätte eine für 34 -fache Vervielfachung ausreichende Platte auch hier zur Verfügung gestanden, so wäre der Vorteil der Minimumstrahl- kennzeichnung noch deutlicher gewesen; doch reicht die Genauigkeit für das gesteckte Ziel ohnehin aus.

\section{Diskussion der Fehlerquellen}

Diese kann aus Platzgründen nur als stichwortartiger Auszug aus den Ergebnissen veröffentlicht werden. Die Angaben beziehen sich auf $D / \lambda$.

Beiträge zur Doppelwertsbreite der Minima: Inkohärenz durch Breite des Lichtquellenspaltes: 0,6; Inkohärenz durch spektrale Breite 0,07; Minimumverschmierung durch Reflexion am Silber 0,05; Lichthof und Silberkorngröße 0,4. Resultierende Minimumbreite 0,75. Da bei ausreichender Vergrößerung im Komparator ein Minimum auf $1 / 10$ bis $1 / 5$ seiner Breite reproduzierbar eingestellt werden kann, ist der durch die Minimumbreite bedingte zufällige Fehler in der Größenordnung von 0,1.

Schlängelung der Minima durch unregelmäßiges kohärentes Streulicht und vor allem Mängel der Planparallelplatte: Die Oberflächen der Platte hatten Unregelmäßigkeiten von rd. 0,02 $\mu$ Tiefe; dadurch bedingte Schlängelung der Minima 0,3. Da man bei der Komparatoreinstellung die seltenen größten $\mathrm{Ab}$ weichungen auszuschließen pflegt, kann dieser Fehler auf etwa die Hälfte herabgedrückt werden.

Fehlerhafte Verschiebung der Minima: Durch Beugung an vorderer Ag-Schichtkante leiden die Randminima (in Abb. 8 als Kreuze gekennzeichnet) und sind zur Auswertung nicht heranzuziehen. Verzeichnung durch das Mikroobjektiv bei Abweichung von symmetrischem Strahlengang 0,14 . Wegen häufiger Umjustierung nur als Streuung der Meßpunkte wirksam; doch im Bereich $10 \cdot 10^{-3}<\alpha^{\prime}<15 \cdot 10^{-3}$, in dem nur ein Film für jede Polarisation angefertigt wurde, leichte systematische Verschiebung aller Meßpunkte (um höchstens 0,14) möglich.

Fehler in der Messung des Kreuzungswinkels $\Delta: \Delta$ ist auf $0,8 \%$ unsicher; Auswirkung auf $D / \lambda$ nur nahe Totalreflexionsgrenze etwa 0,2.

Unsicherheit der Einfallswinkel: $\pm 0,2 \cdot 10^{-3}$ Radien; in den flacheren Teilen der Kurven der Abb. 8 verursacht das nur geringe zufällige Fehler; in den steilen Teilen, z. B. bei $\alpha^{\prime}=2 \cdot 10^{-3}$ Radien steigt die Wirkung auf $\pm 0,2$, und in der unmittelbaren Umgebung der Spitze bewirken Winkelfehler durch eine Art Mittelung mit benachbarten Ordinatenwerten eine Abrundung der Spitze.

Addieren wir alle Fehler (wegen ihrer Unabhängigkeit quadratisch), so müssen die Messungen im Totalreflexionsbereich bis auf einen mittleren Fehler der Einzelmessung von 0,3 an $D / \lambda$ als zuverlässig angesehen werden. Eine intervallweise vorgenommene Ausgleichsrechnung ergab einen mittleren Fehler von rund 0,05 bis 0,1 . Da auch die systematischen $\mathrm{Ab}$ - 
weichungen (insbesondere an den Spitzen) ihre zwanglose Erklärung fanden, kann die Übereinstimmung zwischen Experiment und Theorie als vollkommen gelten.

Wenn auch die Mängel der Planparallelplatte nicht die volle Leistungsfähigkeit der Minimumstrahlkennzeichnung ausschöpfen ließen, so kann doch das Problem der Strahlversetzung bei Totalreflexion und insbesondere das der Polarisationsabhängigkeit als geklärt angesehen werden.

\section{Polarisationsabhängigkeit bei der Maximummethode}

Es schien reizvoll, ergänzend auch die Polarisationsabhängigkeit für die Versetzung eines „Strahles“ im Sinne von Goos und Hänchen zu suchen. Inwieweit das Aussicht auf Erfolg hat - zumal unsere Platte nur eine 25-fache statt 34-facher Vervielfachung erlaubt —, ist am klarsten mit dem Begriff der Subtilität zu erfassen, der in der vorhergehenden Arbeit auf S. 143 eingeführt wurde. Sehen wir Gl. (34) auch für die Maximummethode als in groben Zügen richtig an (wobei der Kreuzungswinkel nun als Apertur gedeutet werden muß), so können wir bei Minimummethode und Maximummethode zugleich die Subtilität in folgender Weise abschätzen.

$\sigma_{\mathrm{s}}=\frac{i}{\mathfrak{D}_{\mathrm{s}} \Delta}=\frac{1,15 \pi}{\sqrt{\iota^{\prime}+\Delta}-\sqrt{\epsilon^{\prime}}} \gtrsim \frac{1,15 \pi}{\sqrt{\Delta}} ; \quad \sigma_{\mathrm{p}}=\sigma_{\mathrm{s}} \frac{D_{\mathrm{p}}}{D_{\mathrm{s}}}$.

Der rechts stehende Minimalwert wird nur an der Totalreflexionsgrenze $a^{\prime}=0$ erreicht. In unserem Untersuchungsbereich bis $\alpha^{\prime}=20 \cdot 10^{-3}$ Radien liegt die Subtilität des Effekts zwischen

$$
72 \leqq \sigma_{\mathrm{s}} \leqq 420 ; \quad 31 \leqq \sigma_{\mathrm{p}} \leqq 210 .
$$

Durch Vervielfachung mit $N=25$-facher Totalreflexion ist daher dic Subtilität auf

$$
2,8 \leqq \sigma^{\mathrm{s}} N \leqq 17,3 ; \quad 1,2 \leqq \sigma_{\mathrm{p}} N \leqq 8,8
$$

herabzusetzen. Die Meßbarkeit $\mu$ des Effekts hängt außerdem von der Meßmethode ab; wird deren „Strahlschärfe" mit $g$ bezeichnet, so ist $g=1$ für die Maximummothode und $g=10$ bis $g=25$ bei der Minimummethode. Lie Meßbarkeit $\mu$ ist dann

$$
\mu_{\mathrm{s}}=\frac{g N}{\sigma_{\mathrm{s}}} \approx \frac{N g\left(\sqrt{\left.\iota^{\prime}+\right\lrcorner}=\sqrt{a^{i}}\right)}{1,15 . \tau} \leq \frac{N g \sqrt{\lrcorner}}{1,15 \cdot \pi}
$$

und liegt in unserem Untersuchungsbereich in den Grenzen

$$
\begin{gathered}
0,8<\mu_{\mathrm{s}}<5 ; 1,6<\mu_{\mathrm{p}}<11,5 \\
\text { für Minima der Schärfe } \mathrm{g}=14 \\
0,06<\mu_{\mathrm{s}}<0,36 ; 0,11 \leq \mu_{\mathrm{p}}<0,8 \\
\text { für die Maximummethode. }
\end{gathered}
$$

Da eine Messung des Effekts (auf maximal 10 bis $20 \%$ mittleren Fehler der Einzelmessung) eine Meßbarkeit $\mu \gtrsim 1$ voraussetzt $^{6}$, kann mit der Minimummethode der ganze Bereich ersichtlich gut erfaßt werden, wie es ja geschehen ist; aber auch bei der Maximummethode müßte wenigstens die unmittelbare Nähe der Totalreflexionsgrenze noch einen verläßlichen Nachweis der Versetzung bei senkrechter Polarisation (erst recht natürlich bei paralleler Polarisation) und daher auch der ebenso großen Polarisationsdifferenz erlauben.

Erforderlich ist nach (42) nur, daß die Apertur des „Strahles“ nicht zu klein wird. Da sehr große Aperturen (4) aber nach (34) absolut kleine Versetzung geben, die unter den Unregelmäßigkeiten der Planparallelplatte verschwindet, kommt es auf optimale Wahl der experimentellen Bedingungen an. In der Apparatur nach Abb. 3 wurde der rechts von dem Rechtwinkelprisma liegende Teil durch einen sehr engen (mit polarisiertem Rotfilterlicht beleuchteten) Lichtquellenspalt und einen von ihm $97 \mathrm{~mm}$ entfernten - unmittelbar vor dem Prisma stehenden - strahldefinierenden Spalt ersetzt.

Abb. $9 \mathrm{a}^{*}$ zeigt die Aufnahme (in 8-facher NachvergröBerung) bei einem Spalt schmalsten Beugungsbildes, Abb. $9 b^{*}$ dasselbe bei einem engeren und $9 c^{*}$ bei weiterem Spalt (jeweils links für senkrechte und rechts für parallele Polarisation).

Die Polarisationsabhängigkeit ist deutlich; aber die quantitative Auswertung ist schwierig. Erstens können nur Flanken als Bezugslinien dienen, deren Lage von der Intensität abhängt; Mittelbildung kann das nicht verläßlich ausgleichen, da beide Flanken starken Winkelabstand, also verschiedenes Schicksal hatten. Zweitens werden die rechten Nebenmaxima stark aufgehellt und stören. Drittens ist eine genaue Messung zwischen Einfaliswinkel und Grenzwinkel kaum möglich, da der Grenzwinkel selbst nicht ohne weiteres erkennbar ist (im Gegensatz zur Minimummethode, wo noch außerhalb des strahldefinierenden Minima Licht bis zum Grenzwinkel vorhanden ist).

Der Wert des Maximumverfahrens wird aber besonders gemindert, weil nicht einmal ohne weiteres zu entscheiden ist, ob die Versetzung der linken Kante auf wahrer Strahlversetzung beruht oder durch Intensitätsbeschneidung am Grenzwinkel vorgetäuscht ist. Die beiden Aufnahmen (Abb. 9c) zeigen einen solchen - besonders krassen Fall -, in dem ein sehr breiter Spalt $(0,6 \mathrm{~mm})$ vorlag, der bereits die bekannten Fransen der Fresnelschen Beugung erzeugte und dadurch Aufschluß über die Ursache der starken scheinbaren Versetzung an der linken Kante gab. Offenbar handelt es sich um eine Intensitätsbeschneidung am Grenzwinkel und nicht um wahre Versetzung, da man in dem Bild für senkrechte Polarisation im Bereich partieller Reflexion die wahre Versetzung mit Hilfe der Minima verfolgen kann. Eine konsequente Vervollkommnung dieser Kennzeichnungsmethode führt übrigens wieder zwangsläufig zur Minimumstrahlkennzeichnung.

${ }_{6} \mathrm{Um}$ die Erinnerung an jene Begriffsbildungen zu unterstützen, erwähnen wir, daß $\mu=1$ nach Definition Gleichheit der Versetzung und der Strahlhalbwertsbreite bedeutet. Die „Nachweisgrenze“ liegt unter 1 und hängt von dem Aufwand ab, der zum Nachweis getrieben wird wir können sie entsprechend der Definition unserer früheren Arbeit als bei $\mu=0,2$ liegend ansehen.

* s. Tafel S. 144 a. 
Man beachte, daß die Scheinversetzung natürlich keine Polarisationsabhängigkeit zeigt. Es soll hier jedoch nicht unterstellt werden, daß der Befund von G. u. H., nach dem keine Polarisationsabhängigkeit bestehen soll, auf dieser Fehlerquelle beruht; die hohe Subtilität des Effektes kann bei seiner ersten Entdeckung solche Feinheiten auch aus vielen anderen Gründen leicht übersehen lassen.

Messen wir visuell oder in den Aufnahmen Abb. 9 a und $9 \mathrm{~b}$ das Verhältnis zwischen der Versetzung der rechten Kanten bei verschiedener Polarisation - der Leser kann das wegen der insgesamt 800-fachen Vergrößerung in den Aufnahmen leicht mit einem gewöhnlichen Maßstab kontrollieren - , so erhalten wir ein Polarisationsverhältnis

$$
D_{\mathrm{p}} / D_{\mathrm{s}}=2 \pm 0,5 \text {. }
$$

Die linken Kanten sind durch Beschneidung am Grenzwinkel gefährdet, mindestens bei $9 \mathrm{~b}$ und $9 \mathrm{c}$.

Die Maximummethode liefert also etwa die gleiche Polarisaticnsabhängigkeit wie die Minimummethode. Eine quantitative Untersuchung der Winkelabhängigkeit schien aus den genannten Gründen nicht mehr lohnend.

\section{$\mathrm{Zus}$ a m m e n f a s sung}

1. Werden in einer Welle Ebenen als Nullebenen der Intensität gekennzeichnet, so läßt sich die Minimumversetzung, d. h. die seitliche Versetzung jener Ebenen, bei Reflexion theoretisch streng berechnen und experimentell genauer als bei einem Energiestrahl messen.

2. Eine Minimumversetzung tritt nicht nur bei Totalreflexion auf, sondern auch bei Reflexion an lei- tenden Medien; stets ist sie polarisationsabhängig. Dagegen findet bei gewöhnlicher Reflexion an der Grenze nicht absorbierender Medien keine Versetzung statt.

3. In allen Fällen, außer bei Totalreflexion, tritt eine Verschmierung der Minima ein.

4. Experiment und Theorie geben übereinstimmend für die Totalreflexion an einer Glas-Luft-Grenze eine Abhängigkeit der Strahlversetzung vom Einfallswinkel nach Art der Abb. 4. Eine unendlich große Strahlversetzung am Grenzwinkel, wie eine vereinfachte Theorie sie vorhergesagt hatte, ist wegen der notwendig endlichen Apertur eines Strahles nicht reell.

5. Die Versetzung der Minimumebenen ergibt sich aus dem Experiment und aus der Theorie übereinstimmend für parallele Polarisation ( $\xi \|$ Einfallsebene) bis zu 2,3-mal so groß wie für senkrechte Polarisation.

6. Die Strahlversetzung kann bei Ausblendung des Strahles mit zwei Spalten wegen der großen Subtilität des Effektes und wegen der prinzipiell auf diese Weise nicht beliebig herabzusetzenden Strahlbreite nicht genau gemessen werden; doch konnte auch hier noch eine Polarisationsabhängigkeit von etwa derselben Art und Größe wie bei der Minimumstrahlkennzeichnung experimentell nachgewiesen werden.

Hrn. Prof. Dr. Lochte-Holtgreven dankt der Verf. für die Unterstützung seiner Arbeit.

\title{
Über eine Beobachtungskammer für ultramikroskopische Untersuchungen an Aerosolen
}

\author{
Von Otтo-Erich SchweckendieK * \\ (Z. Naturforschg. 5 a, 153-156 [1950]; eingegangen am 26. Oktober 1949)
}

\begin{abstract}
Es wird eine Kammer für ultramikroskopische Untersuchungen an Aerosolen beschrieben. Die Radien der Schwebestoffteilchen werden aus den photographierten Fallstrecken der Teilchen im Erdschwerefeld ermittelt. Einige auf diese Weise gewonnenen Teilchengrößen-Verteilungskurven werden wiedergegeben.
\end{abstract}

D ie Kenntnis der Teilchengröße und ihrer Verteilung in einem Aerosol zu verschiedenen Zeiten nach dessen Herstellung ist für die Beurteilung des Aerosols (Alterung, Stabilität, Schwebefähigkeit) von ausschlaggebender Bedeutung. Für die Aufstellung einer Verteilungskurve für die Teilchengröße sind mehrere hundert Einzelbestimmungen notwendig.

Der Radius eines kugelförmigen Aerosolteilchens läßt sich durch die Bestimmung seiner Fallgeschwindigkeit nach der Stokesschen Formel ${ }^{1}$ ermitteln. Es gilt für ein kugelförmiges Teilchen mit dem Radius $r$ und der Dichte $\varrho$, welches mit der konstanten Geschwindigkeit $v$ in einem Medium mit der Dichte $\varrho^{\prime}$ und der inneren Reibung $\eta$ fällt, das Stokesche Gesetz: Gewicht - Auftrieb $=$ Reibungskraft ${ }^{4} / 3 r^{3} \pi g\left(\varrho-\varrho^{\prime}\right)$ $=6 \pi r \eta v$, woraus sich der Radius $r=3 \sqrt{\frac{\eta v}{2 g\left(0-\varrho^{\prime}\right)}}$ ergibt.

- Eystrup a.d. Weser, Bahnhofstr. 161.

1 G. S tokes, Trans. Cambridge Philos. Soc. 9, $5-8$ [1856]. 